\title{
Pouvoir et territoire en Russie
}

\section{Marie Mendras}

\section{(2) OpenEdition \\ Journals}

\section{Édition électronique}

URL : http://journals.openedition.org/conflits/265

DOI : $10.4000 /$ conflits.265

ISSN : $1777-5345$

Éditeur :

CCLS - Centre d'études sur les conflits lilberté et sécurité, L'Harmattan

\section{Édition imprimée}

Date de publication : 15 mai 1996

ISSN : 1157-996X

\section{Référence électronique}

Marie Mendras, "Pouvoir et territoire en Russie », Cultures \& Conflits [En ligne], $21-22$ I printemps-été 1996, mis en ligne le 15 mars 2006, consulté le 30 mars 2021. URL : http://journals.openedition.org/ conflits/265; DOI : https://doi.org/10.4000/conflits.265

Ce document a été généré automatiquement le 30 mars 2021.

Creative Commons License 


\title{
Pouvoir et territoire en Russie
}

\author{
Marie Mendras
}

1 L'histoire de la fin de l'U.R.S.S. montre qu'il est plus difficile de sortir d'une longue histoire impériale que de s'affranchir de l'idéologie communiste ou de l'économie centralisée. Voir se désagréger un régime prédateur et l'appareil du Parti communiste n'a pas été le plus traumatisant. Dans les années quatre-vingt, peu de Soviétiques s'identifiaient encore au régime communiste, un système de gouvernement dont ils cherchaient à se protéger ou à en profiter. La désaffection des Soviétiques à l'égard de l'idéologie et du régime dirigeant n'avait cessé de croitre depuis les années 1960. Les valeurs communistes et les grands dogmes de l'organisation de la société étaient profondément fissurés bien avant la glasnost, le parler-vrai de Mikhaïl Gorbatchev. Le gouverné comme le gouvernant contournaient le système. Les privilégiés du régime avaient déjà "humanisé » l'économie, par la corruption et les lois des réseaux. La société, loin d'être une société civile, était devenue beaucoup moins malléable. Une forme de « résistance » par le moindre effort et l'évanouissement de l'intérêt public, du bien commun, minaient de l'intérieur un État productiviste. En revanche, l'U.R.S.S. était, pour la majorité des Soviétiques, leur État, un ensemble de quelque 300 millions d'individus qui partageaient le même destin depuis des décennies, le plus souvent depuis des siècles. Le pays impérial, puis soviétique, était un espace construit, intégré dans les mentalités, avec quelques exceptions importantes (notamment les Tchétchènes et les autres peuples déportés par Staline dans les années 1940, et les pays Baltes). Les autres nationalités avaient aménagé une double appartenance à leur République ou territoire national et à l'U.R.S.S.. L'idéologie officielle, impressionnante déformation des réalités, avait enraciné une vision de Soi et de l'Autre - le monde extérieur, l'occident capitaliste- commode car simplificatrice : encerclé par un monde hostile, on était d'abord Soviétique. Ainsi, la décomposition du pouvoir central pendant les années Gorbatchev prend une tout autre dimension quand il ne s'agit plus de casser le monopole du parti communiste mais de voir se fissurer l'espace, le pays soviétique. D'un côté, la «décommunisation » formelle du régime n'a pas empêché une grande permanence des élites politiques et économiques, dans presque toutes les républiques ex-soviétiques. Ceci est particulièrement vrai en Russie, en Ukraine, en Biélorussie, en Asie centrale. D'un autre côté, la «désoviétisation» de l'État a provoqué une rupture 
beaucoup plus décisive. L'État s'est de jure et de facto désintégré ; l'U.R.S.S. a physiquement disparu, le territoire s'est transformé, découpé en quinze États, et surtout a changé de nature. En effet, quand les quinze Républiques fédérées de l'U.R.S.S. obtiennent la reconnaissance de la communauté internationale en 1991, elles gagnent à l'instant deux qualités inespérées : le Démocratique et le National. Issues du démembrement d'un État impérial et antidémocratique, leur indépendance en ferait ipso facto des «États-nations" démocratiques. Telle a été, dans l'ensemble, la compréhension des événements en Occident. Même si de nombreux doutes subsistaient sur la capacité des élites républicaines à gouverner efficacement et démocratiquement, on accordait aux nouveaux États le bénéfice du doute. Tous devenaient des partenaires au sein des institutions internationales et dans certaines institutions régionales, comme la C.S.C.E.. La Russie recevait un traitement privilégié car on reconnaissait sa puissance (territoire, population, ressources, capacité militaire) et on attribuait au nouvel État russe les qualités que l'on louait chez son président Boris Eltsine, démocrate victorieux des communistes après le putsch manqué d'août 1991. La Fédération de Russie était donc démocratique et russe, par opposition, c'est à dire àsoviétique/communiste et à-non-russe, c'est-à-dire Ukrainienne, Arménienne ou Tatare. L'histoire des quatre dernières années montre que les complexités de l'histoire et de l'organisation des territoires et des populations en Russie empêchent la mutation rapide d'un grand morceau de l'ancien empire, la Russie, en un «État-nation » aux contours strictement définis, en une Fédération démocratique, en une " communauté de citoyens " ${ }^{1}$. Espace et puissance Alors que les autres républiques de l'ex-U.R.S.S. ont gagné leur existence politique et juridique, la Russie a perdu territoires, populations, puissance. Elle rassemble aujourd'hui les trois-quarts du territoire soviétique et la moitié de la population soviétique. Elle a conservé la majeure partie des matières premières (en particulier énergétiques) des industries et de l'armée. Elle a, en revanche, perdu des têtes de pont vers l'Europe et vers le Sud, et des voies de communication. Elle s'est surtout déplacée vers l'Est, avec la perte de l'empire extérieur (l'Europe centrale satellisée) et de la partie occidentale de l'empire intérieur (pays Baltes, Ukraine, Biélorussie, Moldavie, républiques caucasiennes). Moscou n'est plus au centre physique de l'ensemble, mais à seulement quelques centaines de kilomètres de la nouvelle frontière occidentale de la Russie. Car toute l'ambiguïté est bien que la Russie, avant 1991, c'était l'U.R.S.S. et le camp socialiste, après 1991 c'est un autre espace, plus petit et moins central sur le continent. La République soviétique de Russie (R.S.F.S.R.) n'avait qu'une timide existence administrative dans le système soviétique. En aucune manière, la Russie de Boris Eltsine ne peut être considérée comme la renaissance d'une "petite» Russie enserrée malgré elle dans le Tout soviétique $^{2}$. La fusion de l'identité russe avec l'identité soviétique avait retiré à la République soviétique de Russie toute possibilité de se donner un caractère «national ». Les Russes ne voyaient pas dans la R.S.F.S.R. leur espace national ou leur patrie. Ce n'était pas la «Communauté imaginée» des Russes ${ }^{3}$. Le territoire a fait l'histoire russe. L'État s'est construit en suivant les mouvements de conquête des terres et en cherchant à affirmer le pouvoir du centre politique et de son administration sur les nouveaux espaces. L'historien Richard Pipes rappelle combien le pouvoir politique et administratif a toujours été en retard sur l'occupation des terres par les paysans, les chercheurs de vison et d'or, les aventuriers, l'armée ${ }^{4}$ La puissance, que le pouvoir tsariste puis soviétique comprend comme la condition nécessaire à l'existence, se mesure d'abord en espace, terres, ressources. Ensuite seulement se pose la question de 
l'administration du territoire et des populations. L'immensité fait partie du paysage russe et prime sur les valeurs de bonne gestion, de rentabilité, de règles et de limites. A partir du XVIIIe siècle, les questions de gestion des territoires vont se poser de manière de plus en plus aiguë. L'empire s'est étendu jusqu'aux vieilles nations du Caucase et aux peuples musulmans d'Asie centrale. Les allogènes constituent une part croissante de la population. L'immensité implique aussi la diversité des hommes, des climats, des modes de vie. Le pouvoir tsariste n'a pas une politique bien définie pour gérer la diversité. Balançant entre tentatives d'homogénéisation et aménagement des particularismes, il laisse s'affaiblir l'idée "nationale-impériale», construite autour de la triade " Autocratie-Orthodoxie-Narodnost». Le terme «narodnost »est difficile à traduire. Il implique l'idée de la communauté d'un peuple, organisée donc autour du souverain. La réponse du pouvoir soviétique, après la reconquête de l'empire dans les années vingts, sera une politique double, et cynique: la combinaison d'une territorialisation des nationalités dans des républiques et territoires nationaux, avec la « soviétisation » de l'ensemble politique et de chaque individu, nouveau sujet d'un nouveau pouvoir autoritaire. La gestion de l'immensité et de la diversité s'est accompagnée, à chaque période, de l'enfermement de la population sur son espace et de la fermeture du territoire à l'influence étrangère. L'essai de construction d'un tout passait par le nonchoix des parties et l'impossibilité de l'évasion ou de la convergence avec d'autres espaces, d'autres hommes, indépendants du pouvoir russe. L'ennemi capitaliste a été, des années vingts aux années quatre-vingts, diabolisé en un tout hostile et menaçant, personnifié dans les caricatures soviétiques par le spéculateur américain avec des dollars à la place des yeux. Le monde était simple. Il se divisait entre nous, les bons, et eux, les méchants. L'ennemi totalisant permettait au Tout soviétique d'exister. D'autres ennemis existaient, notamment la Chine, mais ils étaient moins centraux dans la vision dichotomique du monde. La disparition brutale de l'Ennemi il y a quelques années est indissociable de l'histoire de la fin de l'U.R.S.S.. Elle est à la fois cause et effet de l'effondrement de l'espace politique et économique soviétique. La disparition de la menace extérieure, enracinée depuis 1917 comme la menace d'annihilation du système soviétique ${ }^{5}$, a privé le système de sa colonne vertébrale. La construction forcée du tout, la répression des particularismes, la centralisation du pouvoir étaient «imposées par les conditions objectives ", c'est-à-dire le danger extérieur. La société devait servir sa défense avant de servir ses propres intérêts de communauté d'individus. Il fallait produire contre l'Autre, non pas produire pour consommer pour Soi. La Russie est, pour la première fois depuis la Seconde guerre mondiale, obligée de s'interroger sur ce qu'elle est, et non plus sur ce qu'est l'Autre, l'Ennemi, et comment le contrer. Elle doit désormais se définir en positif et non en négatif ou en opposition. La campagne pour les élections législatives de décembre 1995 a montré la carence des élites politiques, la pauvreté des propositions crédibles pour le développement économique et social. Aucune formation ou bloc électoral n'a formulé, et chiffré, un projet de société. Le vote protestataire, qui a été massif le 17 décembre 1995, a bénéficié en premier lieu au Parti communiste et au parti extrémiste de Vladimir Jirinovski, tous deux également indigents en matière économique et sociale mais qui exploitent sans scrupule le marasme général et le désarroi de la population. Le thème de l'intérêt national et de la perte de puissance a bien sûr été largement utilisé pendant cette campagne, et par tous les candidats avec des intensités diverses. Le débat prend facilement un ton revanchard. La défense de l'espace et de la puissance nourrit un ressentiment à l'égard de ceux qui ont contribué à casser le bel ensemble construit depuis des siècles par le 
pouvoir russe. Sont également coupables Mikhaïl Gorbatchev, les économistes libéraux, l'Occident qui voulait «en découdre avec la puissance communiste », et aujourd'hui aussi Boris Eltsine qui, en jouant l'identité russe contre la carte soviétique en 1990-1991, aurait précipité la fin de l'U.R.S.S.. Les termes du débat en Russie, plus de quatre années après la fin de l'U.R.S.S., montrent combien est douloureuse la conversion d'une idéologie de la puissance territoriale et militaire en une idée de l'État «moderne et raisonnable», d'abord soucieux du bien-être et de la sécurité de sa population. Responsables politiques et experts à Moscou aiment rappeler les faiblesses structurelles des nouveaux États: l'Ukraine n'a pas d'indépendance énergétique, la Biélorussie n'a pas d'histoire nationale, les États baltes sont très petits, les États du Caucase sont minés par les conflits, les pays d'Asie centrale sont si pauvres... et peuplés de musulmans. Dans ces conditions, comment ne pas s'attendre à ce que tous ces anciens territoires soviétiques demandent la "réintégration» dans une structure commune autour de la Russie ? L'union fait la force, disent les Russes. L'immensité et le Diktat de Moscou ne nous ont rien apporté de bon, répondent les nouvelles capitales indépendantes. Jusqu'à présent, la Russie n'a pas proposé à ses anciennes républiques une politique de coopération "post-coloniale » attractive. Peut-être par manque de liquidités, peut-être par manque de réflexion à long terme ? L'obstacle principal à une redéfinition des relations avec les pays voisins réside encore dans le refus des nouvelles souverainetés. Même si on ressent confusément à Moscou que l'espace n'est plus la source de la puissance, on ne s'est pas réconcilié avec la perte du contrôle direct sur les territoires, populations et ressources des républiques et on n'est pas encore prêt à penser en termes de partenariat économique et sécuritaire. Ce qui est profondément déroutant pour les Russes - et ce sentiment me paraît partagé par les politiques, l'intelligentsia et la société - c'est que l'empire s'est perdu dans des élections libres, dans la "démocratisation» voulue par Mikhaïl Gorbatchev. Une construction aussi monumentale, fruit de quatre siècles d'efforts, s'est cassée sous le coup d'une timide introduction du scrutin libre et secret. Les années 1989-1991 vont produire une série d'événements tout à fait étonnants qui renversent les chronologies habituelles, l'ordre des choses auquel nous sommes habitués. Gorbatchev tente d'apporter les prémices de «la communauté des citoyens » sur un ensemble hybride, qui n'est plus un empire du XIXe siècle, n'est pas une vraie fédération, n'est pas non plus un État uniforme et monolithique. Il recherche dans l'instrument de l'élection libre le moyen de modifier un équilibre politique, surtout au sein de la Nomenclature communiste, pour apporter une participation de l'individu qui deviendrait ainsi citoyen. Or, cette réforme intervenait alors même que la " communauté », c'est-à-dire l'espace politique réel était déjà en décomposition. Et la toute nouvelle " communauté » de citoyens, encore dans les limbes, éclatait en quinze communautés potentielles. L'élection libre émergeait avant que les bases de la construction d'une communauté politique démocratique fussent en place.

2 Pouvoir central ou pouvoir fédéral ? La constitution de décembre 1993 n'a pas refondu la Fédération de Russie. Elle l'a simplement reconduite avec la complexité des découpages territoriaux de l'ancienne république soviétique de Russie (R.S.F.S.R.). Selon Arkadi Vaksberg, on ne peut pas dire que les Bolcheviks aient inventé l'idée fédérale en Russie. Avant 1917, l'empire était un patchwork de goubernii et de protectorats, et l'uniformité n'a jamais existé, ni même été un objectif́. Bien sûr, ceci n'en faisait pas une fédération, mais c'était une organisation territoriale et politique diversifiée. Avec les années 20 et 30, cet ensemble prend la forme " constitutionnelle » 
d'une Fédération, avec droit de sécession pour les républiques, mais le projet est uniformisateur et totalitaire. L'idée soviétique n'est pas fédérale et, sous couvert de fédération, casse l'esprit de rassemblement de la Russie tsariste. La fédéralisation formelle a pour objet de tuer le Fédéralisme, et même toute idée d'autonomie régionale. Les diversités régionales s'affirmeront à partir des années 1960. Les raisons en sont surtout économiques car les élites républicaines et régionales avaient intérêt à faire de leur espace administratif un espace de pouvoir économique et une base d'enrichissement personnel. Le statut particulier des républiques nationales, hier des «Républiques autonomes » de la R.S.F.S.R., complique la question fédérale. Même si la souveraineté de ces territoires n'est pas reconnue dans la constitution du 12 décembre 1993, leur qualité d'État (Gosudarstvo) est inscrite ainsi que leur droit à une Constitution républicaine et à un président, dans la plupart des cas élu au suffrage universel direct. Si l'on ajoute aux 21 républiques les 10 districts nationaux, la Fédération comprend 31 «sujets » ou membres qui portent le nom d'un peuple autre que russe. Même si ces territoires ne rassemblent qu'une petite partie des 150 millions d'habitants de la Russie, leur particularité les distingue des régions et territoires russes qui portent le nom de leur capitale. L'identification à la république est en règle générale plus forte que l'identification à la région administrative, même pour les Russes qui vivent dans une république nationale comme le Tatarstan ou la Bouriatie. Le rapport à l'ensemble fédéral, qu'on continue à appeler «le Centre» ou le pouvoir central, varie d'une région à l'autre, d'une république à l'autre, en fonction des ressources propres du territoire et de la force des revendications identitaires. La guerre en Tchétchénie a démontré de manière dramatique que l'incapacité à gérer pacifiquement une situation particulière ébranle les fondements déjà fragiles d'une Fédération héritée des découpages soviétiques. Le pouvoir russe a voulu croire que la question tchétchène serait facilement résolue. On aime bien dire à Moscou que les peuples du Caucase sont devenus au fil des ans des «Soviétiques » comme les autres et que leur identité, leur fierté nationale est artificiellement construite par le général Doudaev. La population ne rêverait que de paix, même si c'est une Pax russica. Il est fort probable que les responsables des Services de sécurité, du ministère de l'Intérieur et quelques hommes politiques ont largement sous-estimé la détermination de la population tchétchène à ne pas se laisser une nouvelle fois écraser par les Russes. Les Tchétchènes, plus encore que les autres petits peuples du Caucase, ont toujours donné beaucoup de fil à retordre au pouvoir colonisateur russe. Ils ont résisté pendant la première moitié du XIXe siècle, de nouveau au lendemain de la Révolution d'octobre 1917, encore une fois pendant la Seconde guerre mondiale. Staline les a matés sans état d'âme : ils ont été déportés par centaines de milliers au Kazakhstan, un beau jour de 1944. La Russie eltsinienne a semble-t-il oublié un peu vite l'histoire de l'U.R.S.S.. Elle n'a pas non plus tiré les leçons de la défaite militaire en Afghanistan. Avec les chars, l'artillerie et les bombardements aveugles, les Russes sont redevenus l'ennemi et l'occupant alors qu'à Grozny, Russes et Tchétchènes cohabitaient pacifiquement depuis des décennies. La Fédération de Russie compte maintenant en son sein des centaines de milliers de réfugiés, russes et tchétchènes. Qui peut prétendre sérieusement aujourd'hui réintégrer les Tchétchènes dans la Russie ? Si l'on entend par Tchétchénie non pas le territoire mais la population qui y vivait avant la guerre, l'échec de la politique moscovite est patent. Les Russes de Tchétchénie sont désormais des réfugiés dans d'autres régions du Caucase du Nord. Quant aux Tchétchènes, ils sont bien décidés à séparer pour longtemps leur destinée de celle de la Russie. Quant à l'argument de 
préserver à tout prix l'intégrité territoriale de la Fédération, quel État véritablement fédéral et démocratique peut-on bâtir sur une guerre ? Le recours à la force ébranle les structures fédérales et les institutions centrales. Alors que Moscou avait réussi à sauver la République soviétique fédérative de Russie du naufrage soviétique en 1991, le pouvoir a déstabilisé un équilibre fragile en stigmatisant la Tchétchénie. Le secret de cette continuité de la construction russo-soviétique tenait précisément à la tolérance de quelques cas particuliers, comme l'indépendance des Tchétchènes, les ambitions du président tatar, la corruption et «franchisation »de têtes de pont, telles SaintPétersbourg et Vladivostok. La nouvelle Constitution fédérale adoptée en décembre 1993 ne signifie pas qu'une refondation de la Fédération (ex-R.S.F.S.R.) ait eu lieu sur une base consensuelle et démocratique. La Tchétchénie, se considérant hors de la Russie depuis 1991, n'avait pas organisé sur son territoire le référendum du 12 décembre 1993. Son inclusion dans une république russo-soviétique, et auparavant dans l'empire russe, n'avait jamais été de son gré. On sait que d'autres républiques et régions ont refusé cette Constitution (par une forte abstention ou un vote contre). Le temps est révolu où un pouvoir central pouvait «s'approprier » un territoire, avec ses populations et ses ressources. L'administration centrale ne peut que proposer des modes de gestion, si elle sert des politiques démocratiques menées par des gouvernants légitimes et reconnus comme tels par l'ensemble des territoires qui constituent l'État fédéral. Il y a un long chemin à parcourir avant que les habitants de la Russie ne parlent d'autorités fédérales plutôt que du pouvoir central. Les frontières peuvent être "dépassées", relativisées, quand elles sont le contour net d'un espace clairement défini et reconnu par tous ceux qui y habitent. Il faut également que ce territoire soit administré selon des règles claires pour que le pays puisse faire face aux exigences de l'International. Plus le pouvoir russe entretient le flou sur son État-territoire, plus la « Nation-unité politique » est insaisissable ou sujette à controverses, plus la Russie est tentée, de nouveau, par le repli sur soi et sur sa "spécificité ». Le territoire est une donnée absolue, délimitée par des frontières. Cependant, pris seul, le territoire est un désert. Un espace vit par ses populations, ses ressources, ses communications et c'est par elles que le territoire acquiert une cohérence interne. Le raccourci est peut-être abrupt mais l'histoire russe des cinq dernières années est l'histoire d'un État qui s'invente sans territoire, sans espace politique défini, alors que les élites au pouvoir entretiennent le flou sur les contours et la nature de l'État. En apparence, tout est clair : une fédération, des institutions démocratiques, les frontières de l'ancienne R.S.F.S.R.. Or, en réalité, la Russie doit impérieusement aujourd'hui se donner un territoire, un espace politique, sécuritaire, économique, social, bref un territoire national. Il ne suffit pas de régner, il faut gouverner. Malgré cela, les dirigeants tendent à privilégier le flou - porte ouverte sur des reprises de territoires, d'influence - sur la définition claire des limites, des contraintes, des conditions de la bonne gestion. Ceci est d'autant plus déroutant aujourd'hui que le phénomène électoral a depuis lors perdu sa vitalité et paraît une innovation presque dérisoire aux yeux de beaucoup de Russes qui retiennent d'abord la dureté des conditions de vie et la corruption. 


\section{NOTES}

1. Dominique Schnapper, La communauté des citoyens. Sur l'idée moderne de nation, Gallimard, Paris, 1994.

2. Cf. Marie Mendras, « Existe-t-il un État russe ? », Politique étrangère, 1/92, pp. 25-34 ; Marie Mendras (dir.), Un État pour la Russie, Bruxelles, Complexe, 1992.

3. Cf. Marie Mendras, « Existe-t-il un État russe ? ", Politique étrangère, 1/92, pp. 25-34 ; Marie Mendras (dir.), Un État pour la Russie, Bruxelles, Complexe, 1992.

4. Richard Pipes, Russia under the Old Regime, London, Penguin, 1977.

5. Cf. Nathan Leites, The Operational Code of the Politburo, McGraw-Hill, New York, 1951, et A Study of Bolshevism, Glencoe (Illinois), The Free Press.

6. Arkadi Vaksberg, « Oubiistvennaia igra », Literatournaia Gazeta, 25 janvier 1995.

INDEX

Mots-clés : Etat-nation, territoire(s) et territorialité, politique

Index géographique : Russie 